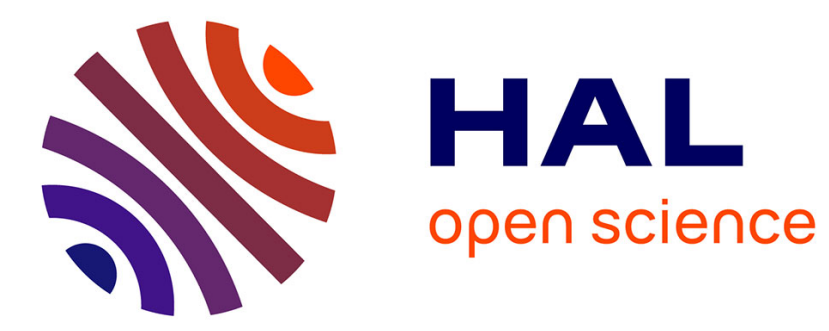

\title{
Sonic boom in soft materials: The elastic Cerenkov effect
} Jeremy Bercoff, Mickaël Tanter, Mathias Fink

\section{To cite this version:}

Jeremy Bercoff, Mickaël Tanter, Mathias Fink. Sonic boom in soft materials: The elastic Cerenkov effect. Applied Physics Letters, 2004, 84 (12), pp.2202-2204. 10.1063/1.1667613 . hal-02481731

\section{HAL Id: hal-02481731 \\ https://hal.science/hal-02481731}

Submitted on 17 Feb 2020

HAL is a multi-disciplinary open access archive for the deposit and dissemination of scientific research documents, whether they are published or not. The documents may come from teaching and research institutions in France or abroad, or from public or private research centers.
L'archive ouverte pluridisciplinaire HAL, est destinée au dépôt et à la diffusion de documents scientifiques de niveau recherche, publiés ou non, émanant des établissements d'enseignement et de recherche français ou étrangers, des laboratoires publics ou privés. 


\title{
Sonic boom in soft materials: The elastic Cerenkov effect
}

\author{
Jeremy Bercoff, Mickaël Tanter, and Mathias Fink \\ Laboratoire Ondes et Acoustique, ESPCI, University Paris VII, CNRS UMR 7587, \\ 10 rue Vauquelin 75005 Paris, France
}

(Received 7 October 2003; accepted 12 January 2004)

\begin{abstract}
We report an experimental evidence of an elastic sonic boom in soft materials. Our approach is based on the ultrasonic remote generation, inside soft media, of a supersonic moving source radiating shear waves in a Mach cone. In analogy with the Cerenkov electromagnetic radiation emitted by a beam of charged particles moving at a speed greater than the speed of light, an intense shear wave is radiated in soft materials. Such Mach waves are sensitive to medium elasticity inhomogeneities and are of great interest for ultrasound-based medical imaging applications. (C) 2004 American Institute of Physics. [DOI: 10.1063/1.1667613]
\end{abstract}

In seismology, compressional $(P)$ and shear $(S)$ waves are commonly used to map the structure of the earth. In soft materials, i.e., the human body, one usually uses arrays of piezoelectric transducers that transmit and receive only ultrasonic $P$ waves in the MHz range to map the organs. In this frequency range, $S$ waves cannot propagate due to very high absorption induced by shear viscosity. However, at sonic frequencies, such $S$ waves can propagate over long distances in soft materials and tissues. We report in this letter a technique allowing the remote generation of a mechanical supersonic source radiating shear waves in soft solids. This effect is the analog of the Cerenkov electromagnetic radiation ${ }^{1}$ emitted by a beam of high-energy charged particles passing through a transparent medium at a speed greater than the speed of light in that medium. This well-known effect is used to detect cosmic rays passing through the atmosphere. In our experiment, the mechanical moving source of vibration is created remotely with ultrasonic radiation pressure. It generates transient shear waves, initially propagating in a Mach cone, that are progressively distorted by shear tissue inhomogeneities. This Mach cone of shear waves propagating in an elastic soft solid is analogous to the "sonic boom" induced in the air by a supersonic aircraft. Integrating this modality in a new, ultrafast ultrasonic scanner (5000 frames $\mathrm{s}^{-1}$ ) allowed us to follow, with a submillimeter resolution, the motion of these shear waves in a large region of interest. This concept can be used to deduce quantitative maps of the shear modulus in human organs and has a strong potential in medical imaging.

It is well known that focused ultrasonic waves generated by piezoelectric arrays induce, during the insonification time, an acoustic radiation force that can be used to remotely push tissues in the focal zone of the beam. ${ }^{2-4}$ Typically, a $300 \mu \mathrm{s}$ duration burst of ultrasound creates locally a transient volumic force around $3 \mathrm{kHz}$. This force acts as a dipolar source of shear waves mainly radiated in transverse directions. Bipolar shear waves can be modeled in soft tissues by a Green's function. ${ }^{5}$

$$
u_{i}(\mathbf{r}, t)=\frac{1}{4 \pi \rho V_{s}^{2}}\left(\delta_{i j}-\gamma_{i} \gamma_{j}\right) \frac{1}{r} \delta\left(t-\frac{r}{V_{s}}\right),
$$

where $r=|\mathbf{r}|, \gamma_{i}=\partial r / \partial i(i=x, y$, or $z), \delta_{i j}$ is the Kronecker symbol, $\rho$ the mass density, $V_{s}$ the shear wave speed, and $u_{i}$ the components of the mechanical displacement vector. Imaging such waves with an ultrasound system is a challenging problem, as these waves propagate through soft solids (like human organs) in less than a tenth of a second. In order to follow the displacements induced by these waves, an ultrasonic scanner able to reach frame rates of 5000 frames s$^{-1}$ is required (a hundred times faster than conventional scanners). The 128 multichannel system developed for this research reaches such high frame rates. ${ }^{6}$ Unlike conventional scanners that use a sequential mode to build an image, the ultrafast mode is performed by emitting a single ultrasonic pulsed plane wave at a $5000 \mathrm{~Hz}$ pulse repetition rate. Backscattered echoes (called rf data) are stored in individual 2 Mbytes of RAM and then transferred to a computer after the acquisition sequence. Typically, the acquisition of 200 frames at 5000 $\mathrm{Hz}$ lasts $25 \mathrm{~ms}$. Then, the echographic images are obtained by beam-forming the rf data in the computer. Finally, consecutive images are compared using one-dimensional cross correlations in order to map axial displacements (along the ultrasonic beam axis) between each frame. Typically, displacements of a micrometer are measurable by the algorithm. The resulting images consist of a movie of the shear wave displacements. The feasibility of using a conventional transducer array (128 elements, 4.3 MHz) for both generation and ultrafast imaging of mechanical shear waves is experimentally demonstrated in a tissue-mimicking phantom: a single ultrasonic focused beam produced by the array insonifies the medium for $300 \mu \mathrm{s}$ (corresponding to 1320 sinusoidal oscillations). Once the pushing sequence ends, the array enters instantaneously into the ultrafast imaging mode and images the medium at 5000 frames s $^{-1}$ during the shear wave propagation. Figure 1 shows axial displacement images at three different times and clearly emphasizes the dipolar directivity pattern of the wave. Measurements provided by our technique are limited to the axial component, as are most of classical ultrasound based motion tracking techniques (for example, the Doppler techniques).

The displacements associated with this diverging shear wave are weak (a few micrometers) and decrease rapidly with the propagation distance. In order to increase the shear 


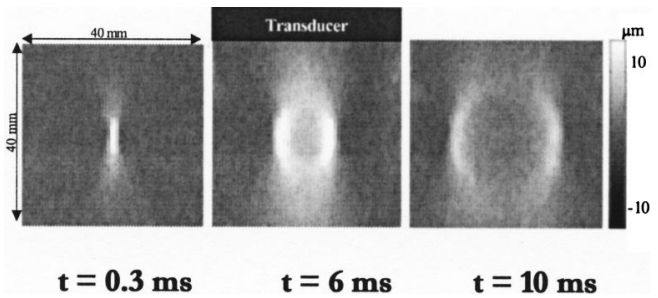

FIG. 1. Ultrafast imaging of the axial displacements induced by a single shear source, created by an ultrasound beam focused in the middle of the imaged area. The experiment was conducted in gelatin-agar elastic phantom. The wave propagates at a $2 \mathrm{~m} / \mathrm{s}$ speed, passing through the medium in $25 \mathrm{~ms}$.

penetration depth and generate high amplitude shear waves in the body, we propose to introduce a supersonic regime. By successively focusing the ultrasonic "pushing" beam at different depths, it is possible to create a moving source radiating shear waves in the body. This can be modeled by taking into account the source movement in Eq. (1):

$$
\begin{aligned}
u_{i}(\mathbf{r}, t)= & \frac{1}{4 \pi \rho V_{s}^{2}}\left(\delta_{i j}-\gamma_{i} \gamma_{j}\right) \\
& \times \iint \frac{q(\tau)}{\left|\mathbf{r}-\mathbf{r}_{0}(t)\right|} \delta\left(t-\tau-\frac{\left|\mathbf{r}-\mathbf{r}_{0}(t)\right|}{V_{s}}\right) d \mathbf{r}_{0} d \tau,
\end{aligned}
$$

where $\mathbf{r}_{0}(t)$ is the moving source location and $q(t)$ is the signal emitted by the source. We can show that in the moving source coordinates, the displacement can be written ${ }^{7}$ as

$$
u_{i}(\mathbf{r}, t)=\frac{1}{4 \pi \rho V_{s}^{2} R \sqrt{1-M^{2} \sin ^{2}(\Theta)}}\left(\delta_{i j}-\gamma_{i} \gamma_{j}\right) f(t),
$$

where $M$ is the Mach number, $R$ the radial distance from the observer to the source, $\Theta$ the angle between the observer's position and the direction of the moving source, and $f$ a function depending on $M, R, V_{s}$, and $\Theta$. If this source is moved faster than the shear propagation velocity, a supersonic regime is established. $M$ is greater than 1 and, as can be seen in Eq. (3), the displacement field is confined in the region $\Theta<\sin ^{-1}(1 / M)$. As a consequence, all resulting shear waves interfere constructively along a Mach cone creating two conical and intense shear wave fronts propagating in opposite directions [Fig. 2(a)]. The angle between both plane waves is proportional to the ratio between the shear wave speed and the speed of the moving source, i.e. to the Mach number $M$. Figure 2(b) shows simulated axial and lateral displacements induced by a Mach 3 supersonic source based on the Green's formalism presented in Eq. (2). Constructive interferences between shear waves create a cumulative effect exclusively along the axial direction making the lateral component negligible. Amplitude displacements can be increased up to $100 \mu \mathrm{m}$ in phantoms and $40 \mu \mathrm{m}$ in human tissues. Moreover, changing the speed of the moving "pushing" beam allows us to change the Mach cone angle. Such versatility enables us to insonify the same medium with different steered plane waves, and gather the same mechanical information from different "points of view." In soft tissues the ultrasonic $P$ wave velocity $\left(1500 \mathrm{~m} \mathrm{~s}^{-1}\right)$ is much higher than the mechanical $S$ wave velocity $\left(1-10 \mathrm{~m} \mathrm{~s}^{-1}\right)$. There-

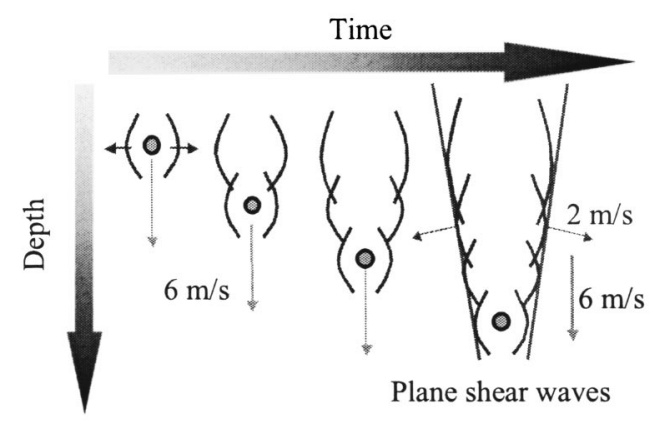

(a)

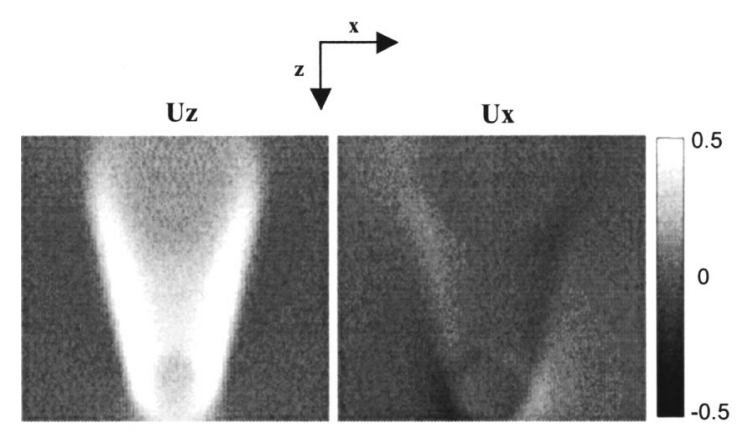

(b)

FIG. 2. The supersonic mode. (a) A shear source activated by ultrasound is moved along the beam axis at supersonic speed. (b) Simulated and normalized axial and lateral displacements at a given time induced by a supersonic regime.

fore, it is possible to create supersonic shear sources moving at incredibly high Mach numbers (up to Mach 1500). The ability to establish a supersonic regime in soft tissues is experimentally demonstrated in a homogeneous phantom. A
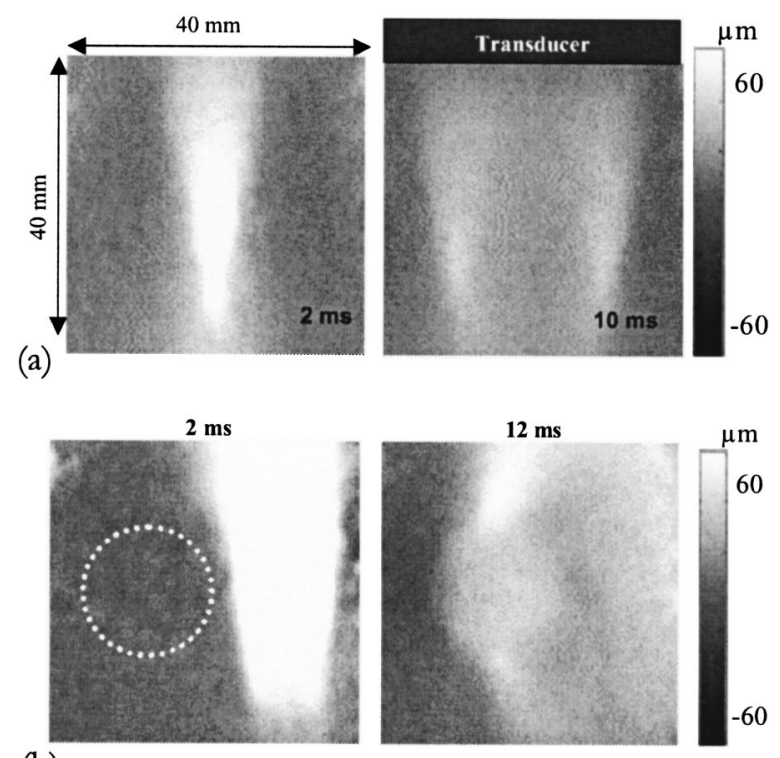

(b)

FIG. 3. The supersonic mode. Experiments conducted in a tissue-mimicking phantom (agar-gelatin): The supersonic mode results in two plane shear waves propagating in a Mach cone (a) whose angle is linked to the shear Mach number (Mach 3). (b) Another Mach 3 supersonic regime, generated on the right of the imaged area, in a heterogeneous phantom containing a 20 mm hard inclusion. 
shear source moving along the beam axis is induced at a supersonic speed. In Fig. 3(a), axial displacements produced by the shear waves are imaged by the ultrafast scanner at two different times. We clearly see them interfering along the Mach cone. In this experiment, the focused "pushing" beam was moved at five successive depths $(8,16,24,32$, and 40 $\mathrm{mm})$ at $6 \mathrm{~m} \mathrm{~s}^{-1}$ speed. As in the simulation above, this particular experiment corresponds to a Mach 3 supersonic regime. The displacement amplitude is higher than in the single push mode $(60 \mu \mathrm{m})$ and the shear wave propagation covers the whole imaged area. Such plane shear waves are of great interest because they are sensitive to the mechanical properties of the tissues. This is illustrated in Fig. 3(b) where a Mach 3 supersonic regime has been generated in a heterogeneous medium containing a harder inclusion. The plane shear wave front is clearly distorted by the medium heterogeneity. This information can be used to recover the elastic parameters of organs and has been applied to human organs, ${ }^{6,89}$ showing the potential applicability of the regime to medical imaging. ${ }^{10}$

Using shear waves activated by the ultrasonic radiation force, it is possible to produce in soft solids the analog of the Cerenkov radiation effect in electromagnetics. The experi- mental evidence of such shear waves propagating in a Mach cone is given in gelatin phantoms using an ultrafast ultrasonic imaging system. This innovation could be achieved with the same ultrasonic probe as the ones used today by physicians. It emphasizes the strong potential of applying this concept to elasticity imaging for breast and liver cancer diagnosis.

${ }^{1}$ J. V. Jelley, Cerenkov Radiation and Its Applications (Pergamon, New York, 1958).

${ }^{2}$ A. P. Sarvazyan, O. V. Rudenko, S. D. Swanson, J. B. Fowlkes, and S. Y. Emelianov, Ultrasound Med. Biol. 20, 1419 (1998).

${ }^{3}$ K. R. Nightingale, R. W. Nightingale, M. Palmeri, and G. E. Trahey, J. Acoust. Soc. Am. 110, 625 (2001).

${ }^{4}$ M. Fatemi and J. F. Greenleaf, Science 280, 82 (1998).

${ }^{5} \mathrm{~K}$. Aki and P. G. Richard, Quantitative Seismology, Theory and Methods (Freeman, San Francisco, 1980), Vol. 1, Chap. 4, p. 74.

${ }^{6}$ L. Sandrin, M. Tanter, S. Catheline, and M. Fink, IEEE Trans. Ultrason. Ferroelectr. Freq. Control 49, 426 (2002).

${ }^{7}$ A. P. Dowling and J. E. Ffowcs Williams, Sound and Sources of Sound (Ellis Horwood, 1983), Chap. 9, p. 187.

${ }^{8}$ J. Bercoff, S. Chaffai, M. Tanter, L. Sandrin, S. Catheline, M. Fink, J. L. Gennisson, and M. Meunier, Ultrasound Med. Biol. 29, 1387 (2003).

${ }^{9}$ R. Muthupillai, D. J. Lomas, P. J. Rossman, J. F. Greenleaf, A. Manduca, and R. L. Ehman, Science 269, 1854 (1995).

${ }^{10}$ J. Bercoff, M. Tanter, and M. Fink, IEEE Trans. Ultrason. Ferroelectr. Freq. Control (to be published). 